Recepción: 12/ 09 / 2018

Aceptación: 10 / 12 / 2018

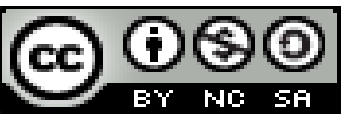

Ciencias económicas y empresariales

Publicación: 20 / 01 / 2019

Artículo de revisión

\title{
El plan de marketing como herramienta de gestión en los procesos de comercialización
}

\section{The marketing plan as a management tool in the marketing processes}

\section{O plano de marketing como ferramenta de gestão nos processos de comercialização}

\author{
Jorge O. Quevedo-Vázquez ${ }^{\mathrm{I}}$ \\ joquevedov@ucacue.edu.ec \\ Lesi V. Giler-Escandón ${ }^{\text {II }}$ \\ lvgilere@ucacue.edu.ec \\ Jorge E. Ormaza-Andrade ${ }^{\text {III }}$ \\ jormaza@ucacue.edu.ec
}

Ramiro O. González-Rodríguez IV

rogonzalezr@ucacue.edu.ec

Manuel R. Quevedo-Barros V

mrquevedob@ucacue.edu.ec

\section{Correspondencia: joquevedov@ucacue.edu.ec}

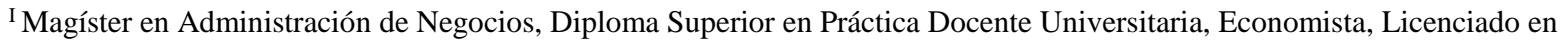
Economía y Finanzas, Subdirección de Posgrados Universidad Católica de Cuenca, Cuenca, Ecuador

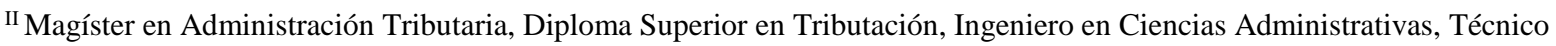
Superior en Administración de Negocios, Subdirección de Posgrados Universidad Católica de Cuenca, Cuenca, Ecuador.

III Magíster en Administración de Empresas Mención en Recursos Humanos y Marketing, Especialista en Docencia Universitaria, Contador Público, Auditor, Ingeniero Empresarial, Universidad Católica de Cuenca, Sede Azogues, Azogues, Ecuador

IV Magíster en Administración de Empresas Mención en Recursos Humanos y Marketing, Contador Público, Auditor, Ingeniero Empresarial, Universidad Católica de Cuenca, Sede Azogues, Azogues, Ecuador
}

\footnotetext{
v Magíster en Contabilidad y Auditoría, Contador Público, Licenciado en Administración, Universidad Católica de Cuenca Sede Azogues, Cuenca, Ecuador
} 


\title{
Resumen
}

El Plan de Marketing en la actualidad constituye una herramienta de alto alcance para la mejora de procesos en el área de comercialización, para ello es necesario contar con informantes claves como: propietarios del negocio y funcionarios que realizan sus actividades gerenciales, toma de decisiones y acciones operativas, por la necesidad de disminuir las debilidades existentes en incrementar las fortalezas en la administración de los recursos financieros y ajustarse a la nueva forma de administrar en base a riegos, normas de solvencia y prudencia. Se parte de un objetivo general que es el de establecer los aspectos que generan el bajo nivel de ventas, así como elaborar un plan de marketing que permita al negocio adecuarse a las necesidades fluctuantes del mercado, generando una ventaja competitiva, de forma que logre incrementar su nivel de ventas, de ello se derivan los objetivos específicos mismos que son: identificar los aspectos administrativos que generan los bajos niveles de ventas, indagar los procesos de comercialización existentes, capacitar al personal, estudiar y analizar a la competencia, diseñar e implementar un plan de marketing, y establecer estrategias de venta para mejorar el sistema de comercialización. En base a los principales hallazgos se diseñó el Plan de Marketing caso Almacén de Pinturas Arco Iris (Quevedo Vázquez, 2013), en base a esta información se realizó la validación de la propuesta con los propietarios del negocio, los cuales evidenciaron apertura y deseo de implantar esta herramienta de gestión que conducirá a la toma de decisiones y acciones correctivas para mejorar su rentabilidad.

Palabras Clave: Competencia; estrategias; marketing; procesos; planificación.

\begin{abstract}
The Marketing Plan today is a tool of high range for process improvement in the marketing area, for it is necessary to have key informants as business owners and officials who perform their managerial activities, decisions and actions operational, by the need to reduce existing weaknesses in increasing strengths in managing financial resources and adjust to the new way to manage based on risks, solvency standards and prudence. It is part of an overall objective is to establish the aspects that generate the low level of sales as well as develop a marketing plan that allows the business to adapt to the changing needs of the market, creating a competitive advantage, so that achieve increase its sales, it specific objectives themselves are derived: identificar administrative aspects
\end{abstract}


that generate low levels of sales, to investigate processes existing marketing, staff training, study and analyze the competition, design and implement a marketing plan, and establish sales strategies to improve the marketing system. Based on the main findings of the Marketing Plan case Paint store Rainbow was designed, based on this information to validate the proposal was made with the owners of the business, which showed openness and willingness to implement this management tool will lead to decision making and corrective actions to improve profitability.

Keywords: Competition, strategies, marketing-, process; planning

\section{Resumo}

O Plano de Marketing na realidade constitui uma ferramenta de alto alcance para a gestão de processos na área de comercialização, para elecesario contar conformantes como: propietários da negocio e dos serviços que realizam as suas actividades, tomadas de decisões e acciones operativas, pela necesidad de disminuir as debilidades existentes em incrementar as fortalezas na administração dos recursos financeiros e no acesso à nova forma de administração na base de um raciocínio, normas de solvencia e prudencia. In the general of the organisations in the plan for marketing that unlock the financial level of the ventas, as such as planning to market that enable a negocio adecuarse a las necesidades fluctuantes del mercado, generando una ventaja competitiva, deforme that logre incrementar the level of sales, de ellse derivate the objectives specified misms that son, such as options administrative, generary the bajos niveles de ventas, indagar the process of commercialisation inexistente, capacitar all personal, estudiar and analizar a la competencia, diseñar e implementar un planejar de marketing, e estabelecer estrategias de vendas para o sistema de comercialização. Em base a los principios hallazgos se diseññan o Plan de Marketing caso Almacén de Pinturas Arco Iris (Quevedo Vázquez, 2013), em base a esta información se realizar a validação da proposição com os propietários do negocio, os cuales evidenciaron apertura y deseo de implantar esta estratégia de gestão que conduz a tomada de decisões e correcções correctivas para maior rentabilidade.

Palabras Clave: Competencia; estrategias; marketing; procesos; planificación.

\section{Introducción}


Las actividades de comercialización en las PYMES, se maneja en forma tradicional, en lo referente al aspecto administrativo y financiero actividades que generalmente se centraliza únicamente en la gerencia, aislándoles a las personas que laboran dentro del negocio de las actualizaciones, políticas y estrategias, lo que genera riesgos en el manejo de liquidez, sostenibilidad, y en el crecimiento institucional.

Para evidenciar este hecho es necesarios conocer que es una Pymes, definiendo el Servicio de Rentas Internas como ( ...) el conjunto de pequeñas y medianas empresas que de acuerdo a su volumen de ventas, capital social, cantidad de trabajadores, y su nivel de producción o activos presentan características propias de este tipo de entidades económicas”. (Servicio de Rentas Internas (SRI), 2016)

Las PYMES en el Ecuador se encuentran en particular en la producción de bienes y servicios, siendo la base del desarrollo social del país en la producción, demanda y oferta de productos, añadiendo valor agregado, por lo que se constituyen en un actor fundamental en la generación de riqueza y empleo.

En la administración de estas empresas los manuales de procesos y procedimientos son desarrollados por la gerencia para las diferentes áreas, quedando pendiente el desarrollo de herramientas de control interno y de riesgos para los responsables de las oficinas por lo que se atribuye la importancia de contar con una herramienta que permita la gestión del proceso de comercialización mediante un plan de marketing.

Un negocio integrante dentro de la Pymes, generalmente es irregular debido a que los procesos administrativos y financieros no son los correctos. Es por ello que requieren contar con un plan de marketing que la conduzca a conocer y satisfacer las necesidades de sus clientes mediante la implementación de estrategias que permitan obtener una ventaja completiva frente a la competencia.

Por lo antes expuesto es necesario plantearnos las siguientes preguntas

¿Qué estrategia de ventas sería la más adecuada para mejorar la situación actual del nivel bajo de ventas de las Pyme caso “ARCO IRIS? (Quevedo Vázquez, 2013)

¿Cuáles son los aspectos administrativos del negocio de mayor incidencia en el bajo nivel de ventas?

¿Cuáles son los procesos actuales de comercialización?

¿Cómo mejorar el desempeño de los empleados? 
¿Analizar quiénes son los competidores a los cuales se enfrenta?

¿Cómo se puede implantar un plan efectivo de ventas y cobros?

¿Cuáles serán los requisitos indispensables para establecer y poner en marcha un plan eficiente de promoción de productos?

\section{Desarrollo}

\section{Justificación de la investigación}

La elaboración de un plan de marketing para las Pymes, se justifica en la medida que permita un desarrollo sostenido y adecuado del negocio, por lo que es necesario identificar las fases que lo componen.

La primera fase de este plan permitirá estructurar las estrategias a seguir con el fin de ampliar su participación en el mercado e incrementar sus ventas y consecuentemente su rentabilidad.

Una segunda fase es conocer ampliamente las características de los consumidores del mercado, con

el objeto de diseñar una apropiada mezcla de marketing; eligiendo adecuadamente el tipo de producto, estableciendo un precio justo, recomendando nuevos sitios de distribución y mejorando la forma de promocionarse.

Una tercera fase consiste en idear un sistema de retroalimentación que permitirá a las Pymes, medir el desempeño del plan, con el fin de la regeneración constante de los elementos que lo conforman, de tal forma, que permita al negocio posicionarse mejor en el mercado.

Los elementos anteriores propone el establecimiento de estrategias que pretendan ayudará a las Pymes a cumplir con sus objetivos, obteniendo una mayor participación en el mercado, brindar un mejor servicio y atención al cliente.

\section{Algunos elementos legales y teóricos a considerar dentro del plan de marketing}

\section{Aspectos legales}

\section{Impuestos}

- Ley Orgánica de Régimen Tributario Interno Este cuerpo legal establece las normas procedimientos, beneficios y sanciones a los sujetos pasivos amparados en este marcojurídico.

- Control de precios Dentro del control de precios se debe seguir los señalamientos establecidos en los artículos 184, 185, 186 de control de precios. 
- Registro de la marca Aunque no es un paso obligado en la creación de una MYPIMES, pero se recomienda registrar las marcas, nombres o lemas comerciales para adquirir los derechos sobre ese nombre especifico y evitar disputas con terceros.

El procedimiento para inscribir una marca comienza con la disponibilidad de ese nombre en el (IEPI), a este procedimiento se le denomina búsqueda y permite al usuario tener conocimiento si una marca, lema o nombre comercial ya fue registrado o está tramitando su inscripción.

Código orgánico organización territorial autonomía descentralización (COOTAD): Se debe tener conocimiento de las diferentes disposiciones y sanciones, puesto que todo negocio requiere de un local dentro de su territorio, el mismo que tiene que pagar tasas prediales, municipales, marcas, patentes, etc. Con el pago de estos impuestos se evitará las diferentes sanciones como multas, clausuras definitivas o clausuras por un tiempo determinado. Para (Acosta, 1995) Impuesto es un pago obligatorio que se efectúa al Estado, ya sea en moneda o especie, sin contrapartida directa, para financiar el gasto público. Por su parte el profesor (de Torres, 2012) Define a la patente como un permiso que proporciona el fisco, por medio del pago de una cuota para ejercer legalmente negocios comerciales o industriales.

Alineando estos dos criterios se entiende al Impuesto de Patente Municipal como un tributo de carácter obligatorio y exclusivo para las personas naturales, personas jurídicas y sociedades, ya sean estas nacionales o extranjeras, que realicen actividades económicas de orden comercial, financiero, industrial, inmobiliario y/o profesionales dentro de los límites territoriales del cantón. Al observar la evolución que ha tenido el Impuesto de Patente en el GAD Municipal de Azogues, se ha encontrado algunos cambios que en su mayoría son reformas a las Ordenanzas del Impuesto de Patente. Desde el año 2011 la gestión y cobro del Impuesto de Patente está a cargo de la Sección de Rentas del GAD Municipal de Azogues, los años anteriores estuvo a cargo de la Sección de Avalúos y Catastros.

- De las rentas patrimoniales: De acuerdo al Código Orgánico de Organización Territorial, COOTAD, en el Art. 491 se manifiesta: “Clases de impuestos municipales. - Sin perjuicio de otros tributos que se hayan creado o que se crearen para la financiación municipal o metropolitana, se considerarán impuestos municipales y metropolitanos los siguientes:

a) El impuesto a sobre la propiedad urbana

Pol. Con. (Edición núm. 29) Vol.4, No 1, enero 2019, pp. 100-115, ISSN: 2550 - 682X, 
b) El impuesto sobre la propiedad rural

c) El impuesto de alcabalas

d) El impuesto sobre los vehículos

e) El impuesto de matrículas y patentes

f) El impuesto a los espectáculos públicos

g) El impuesto a las utilidades en la transferencia de predios urbanos y plusvalía de los mismos

h) El impuesto al juego; e,

i) El impuesto del 1.5 por mil sobre los activos totales

De todos estos nos centraremos en el ítem i) que trata sobre El Impuesto del 1.5 por mil sobre los activos totales.

Art. 552.- Sujeto Activo. - Son sujetos activos de este impuesto las municipalidades y distritos metropolitanos en donde tenga domicilio o sucursales los comerciantes, industriales, financieros, así como los que ejerzan cualquier actividad de orden económico.

Art. 553.- Sujeto Pasivo. - Son sujetos pasivos del impuesto del 1.5 por mil sobre los activos totales, las personas naturales, jurídicas, sociedades nacionales o extranjeras, domiciliadas o con establecimiento en la respectiva jurisdicción municipal, que ejerzan permanentemente actividades económicas y que estén obligados a llevar contabilidad, de acuerdo con lo que dispone la Ley Orgánica de Régimen Tributario Interno y su Reglamento.

Para efectos del cálculo de la base imponible de este impuesto los sujetos pasivos podrán deducirse las obligaciones de hasta un año plazo y los pasivos contingentes.

Los sujetos pasivos que realicen actividades en más de un cantón presentarán la declaración del impuesto en el cantón en donde tenga su domicilio principal, especificando el porcentaje de los ingresos obtenidos en cada uno de los cantones donde tenga sucursales, y en base a dichos porcentajes determinarán el valor del impuesto que corresponde a cada Municipio.

Para el pago de este impuesto por parte de las empresas de prestación de servicios para la exploración y explotación de hidrocarburos, se tendrá en cuenta lo dispuesto en la Ley Orgánica de Régimen Tributario Interno. 
Para la declaración y pago de este impuesto por parte de los sujetos pasivos que tengan actividades permanentes en la provincia de Galápagos se estará a lo dispuesto en la Ley de Régimen Especial para la Conservación y Desarrollo Sustentable de la Provincia de Galápagos.

Cuando los sujetos pasivos de este impuesto tengan su actividad en una jurisdicción distinta al Municipio o Distrito Metropolitano en el que tienen su domicilio social, el impuesto se pagará al Municipio del lugar en donde se encuentre ubicada la fábrica o planta de producción.

Art. 554.- Exenciones. - Están exentos de este impuesto únicamente:

a) El gobierno central, consejos provinciales y regionales, las municipalidades, los distritos metropolitanos, las juntas parroquiales, las entidades de derecho público y las entidades de derecho privado con finalidad social o pública, cuando sus bienes o ingresos se destinen exclusivamente a los mencionados fines y solamente en la parte que se invierta directamente en ellos;

b) Las instituciones o asociaciones de carácter privado, de beneficencia o educación, las corporaciones y fundaciones sin fines de lucro constituidas legalmente, cuando sus bienes o ingresos se destinen exclusivamente a los mencionados fines en la parte que se invierta directamente en ellos;

c) Las empresas multinacionales y las de economía mixta, en la parte que corresponda a los aportes del sector público de los respectivos Estados. En el caso de las empresas de economía mixta, el porcentaje accionario determinará las partes del activo total sujeto al tributo;

d) Las personas naturales que se hallen amparadas exclusivamente en la Ley de Fomento Artesanal y cuenten con el acuerdo interministerial de que trata el artículo décimo tercero de la Ley de Fomento Artesanal;

e) Las personas naturales o jurídicas que se dediquen a la actividad agropecuaria, exclusivamente respecto a los activos totales relacionados directamente con la actividad agropecuaria; y,

f) Las cooperativas de ahorro y crédito.

Para el impuesto sobre el activo total no se reconocen las exoneraciones previstas en leyes especiales, aun cuando sean consideradas de fomento a diversas actividades productivas.

Art. 555.- Plazo para el pago. - El impuesto del 1.5 por mil corresponderá al activo total del año calendario anterior y el período financiero correrá del 1 de enero al 31 de diciembre. Este impuesto se pagará hasta 30 días después de la fecha límite establecida para la declaración del impuesto a la renta". (C. del Ecuador, 2010)

Pol. Con. (Edición núm. 29) Vol.4, No 1, enero 2019, pp. 100-115, ISSN: 2550 - 682X, 
- Código de defensa del consumidor Las disposiciones de la presente ley son de orden público y de interés social, sus normas por tratarse de una ley de carácter orgánico, prevalecerán sobre las disposiciones contenidas en leyes ordinarias. En caso de duda en la interpretación de esta ley, se la aplicará en el sentido más favorable al consumidor.

El objeto de esta ley es normar las relaciones entre proveedores y consumidores promoviendo el conocimiento y protegiendo los derechos de los consumidores y procurando la equidad y la seguridad jurídica en las relaciones entre las partes.

\section{Aspectos teóricos}

\section{La planificación}

Al respecto (Jimenes C, 2004, pág. 25) señala que "la planificación es un proceso de toma de decisiones para alcanzar un futuro deseado, teniendo en cuenta la situación actual y los factores internos y externos que pueden influir en el logro de los objetivos."

La planificación cumple dos propósitos principales en las organizaciones: el protector y el afirmativo. El propósito protector consiste en minimizar el riesgo reduciendo la incertidumbre que rodea a los negocios y definiendo las consecuencias de una acción administrativa determinada. El propósito afirmativo consiste en elevar el nivel de éxito organizacional.

Cuando hablamos de procesos (Terry, 2005, pág. 104) manifiesta "es el proceso de seleccionar información y hacer suposiciones respecto al futuro para formular las actividades necesarias para realizar los objetivos organizacionales."

En el siglo XXI, surge un nuevo modelo de gestión que requiere que las personas deben entender sobre la complejidad de las nuevas tendencias y las deben adoptar.

El nuevo modelo de gestión empresarial gira en torno al CLIENTE FOCUS COSTUMER Los consumidores son la parte más importante de la producción, son los verdaderos artífices del interés de las empresas, esto hace que exista un cambio radical en la filosofía del marketing.

Las compañías deben adoptar diferentes variables y estrategias a las demandas actuales, el consumidor es el que realmente representa la parte más importante de la empresa, deben preocuparse en conocerlo, entenderlo y así poderle dar las respuestas precisas a las necesidades que éste manifieste o consulte.

El departamento de Marketing debe estar impregnado de su filosofía y del saber hacer, en toda la empresa; no se tiene que olvidar en ningún momento que la verdadera razón de ser de la empresa es la entera satisfacción del cliente, lo que conlleva el éxito. 
El acierto de las nuevas tecnologías y la combinación en la era digital de todas las áreas de la organización empresarial le otorgan al marketing estratégico un importante papel como un verdadero conductor de toda la información del negocio y principal actividad para adecuar los productos, procesos y ventas a los gustos y necesidades del cliente. Por lo cual hay que desarrollar las herramientas que nos ayuden a captar el talento y gestionar el conocimiento.

\section{Metodología}

La investigación -Caso Arco Iris- se encuentra bajo el paradigma mixto con datos cualitativos y cuantitativos, por lo tanto incluye elementos positivistas y naturalistas, con investigación descriptiva - exploratoria y propositiva, puesto que los diseños transaccionales descriptivos tiene como objeto indagar la incidencia y los valores en que se manifiestan una o más variables.

Es de tipo descriptivo porque detalla la situación actual del problema y describe claramente sus particularidades y los fallos que tiene por no realizar un plan excelente de marketing, identificando de esta manera todas las deficiencias que tiene la empresa.

Es de tipo exploratoria porque permite familiarizarse con el plan de marketing actual y obtener información para llevar a cabo la investigación, profundizando las causas y efectos del problema de marketing tradicional, que dificultan la toma de decisiones y el desarrollo de actividades a cargo de los responsables del negocio investigado.

La utilización de fuentes secundarias externas como: el INEC, libros, archivos, revistas, Internet, fuentes primarias internas tales como: estados financieros, catálogo de productos, con el propósito de disponer de un panorama mucho más amplio del tema, que permita efectuar un análisis a profundidad.

Además es de tipo propositiva ya que el momento que se obtuvo la información descrita, se realizó una propuesta para superar la problemática actual y las deficiencias encontradas. Se identificó todos los problemas, se investigó, se profundizó y se dio una solución de tipo contextual.

\section{Diseño de la investigación}

Se puedo definir como de tipo no experimental ya que en este tipo de investigación no se manipulan variables; es decir, se observan fenómenos tal y como se dan en su contexto natural para después analizarlos. Este estudio es transversal, ya que se recolecto datos en un solo momento o tiempo único, con el propósito de describir variables y analizar su incidencia e interrelación en un momento dado. 


\section{Variables}

Las variables analíticas utilizadas en la presente investigación son:

- Descriptiva: La gestión tradicional de venta en el negocio "ARCO IRIS", ocasionan dificultades en el crecimiento del negocio, debilitando los ingresos económicos y la toma oportuna de decisiones. Esta variable es cualitativa, mide atributos y cualidades desde el análisis, es exploratoria, extrae información pertinente, describe particularidades del problema y de los elementos asociados.

- Propositiva: Propuesta técnica.- El diseño de un Plan de Marketing, para mejorar las ventas del negocio. Esta variable es cuantitativa, mide y evalúa cantidades, producto de la construcción de información, resultados y experiencia, para solucionar el problema.

\section{Operacionalización de las variables}

Una definición operacional constituye el conjunto de procedimientos que describe las actividades que un observador debe realizar para recibir las impresiones sensoriales, las cuales indican la existencia de un concepto teórico en mayor o menor grado. (Sampieri, Fernández, \& Bautista, 2010, pág. 111). En el caso propuesto - Arco Iris- se consideró las variables de diagnóstico y variables de propuesta.

\section{Variables del diagnóstico}

Dentro de las variables de diagnóstico se consideraron las variables operativas para estudiar el bajo nivel de ventas de las MiPymes, representa los aspectos administrativos, desarrollo del talento humano, y los procesos de comercialización, utilizados en la Pymes.

\section{Variables de la propuesta}

En el estudio a esta variable se definió como: "Se cuenta con los aspectos del plan de marketing, a través de la estrategia de ventas, con promociones de productos; mediante el control interno, basándose en los objetivos estratégicos de ventas del almacén.

\section{Población y muestra}

El universo utilizado para el cálculo de la muestra fue de 210 individuos, distribuidos porcentualmente de la siguiente forma: propietario del negocios $0 \%$, empleados del negocio $2 \%$, clientes 98\%. Como se puede apreciar, la población es mínima, por lo que se aplicó un barrido en razón de que los encuestados fueron de fácil identificación.

\section{Estrategias, técnicas e instrumentos}


- Entrevista: Sirvió para obtener datos de los propietarios del negocio, empleados, maestros, arquitectos, proveedores; con esta información se determinó con claridad cuáles son los objetivos del negocio, estos datos fueron procesados, analizados y se determinó los resultados.

- Encuestas: Fue destinada a los consumidores finales, con esto se obtuvo datos para la investigación, la encuesta fue por escrito y diferente para cada grupo de personas anotado anteriormente; la información fue importante para el análisis y control interno y externo del negocio.

- Observación Directa: Fue revisada con detenimiento, para obtener información en lo que respecta al entorno circulante del negocio, como son cada uno de los productos que distribuye el negocio, estos se plantearon por escrito. Esta observación fue documental y de campo.

- Bibliográfica: Dentro de esta constan los: libros, documentos, manuales, revistas, páginas web, referente al plan de marketing, se analizaron en forma permanente, sobre todo aquellos que ofrecieron información importante.

- Tabulación: Se resumieron los datos en las tablas estadísticas, estas facilitaronla comprensión e interpretación de los datos que se tabularon.

- Análisis de datos: Se realizó el análisis de los resultados, estos dependieron de la complejidad de la hipótesis que se propuso.

- Representación de datos: Se realizó los distintos gráficos estadísticos que existen como pueden ser: barras horizontales, verticales, o a través de los pasteles.

- Interpretación de resultados: Se pudo demostrar que todos los conceptos, destrezas y como éstas se enlazan dentro de la solución del problema, se determinó cuál es la causa del problema y la que más afecta al negocio.

\section{Procedimientos para la investigación descriptiva del problema}

El proceso de investigación que se utilizó fue el siguiente: Análisis del problema propuesto teniendo en claro las causas por las cuales se origina este problema; una vez que se ha definido el problema, se procedió a plantear los objetivos de la investigación lo que permitió tener en claro el que hacer, luego se procedió a plantear las preguntas de investigación o las hipótesis que serán expuestas a comprobación durante todo el proceso investigativo, el siguiente paso es la elaboración del marco teórico, el mismo que busca direccionar la investigación en base a una paradigma que en este caso se plantea el plan de marketing como una alternativa de desarrollo del negocio.

Pol. Con. (Edición núm. 29) Vol.4, No 1, enero 2019, pp. 100-115, ISSN: 2550 - 682X, 
Definido y analizado el marco teórico se procedió a recopilar la información de campo, para lo cual se aplicaron las técnicas e instrumentos definidos, los resultados de esta investigación deben ser procesados y analizados a fin de que exista coherencia de la información levantada, y finalmente a partir de allí se procede a formular las conclusiones y recomendaciones.

\section{Procedimientos para la construcción de la propuesta}

Con la aplicación del nuevo plan de marketing, se pretende mejorar las ventas del negocio, hacerlo conocer más dentro del medio, a través de la publicidad y promociones que aplique el negocio mediante cursos de capacitación para el personal que labora, así como también ofrecer cursos para las personas que consumen en el local; viendo a trabajadores y consumidores finales, como seres humanos con destrezas y habilidades; llevar un manejo correcto del control interno, administrativo, técnico y profesional dentro del nuevo diseño del plan de marketing.

Fue necesario realizar una socialización sobre los cambios que se desean realizar para mejorar la presentación del negocio.

Con todos estos resultados la información se tabulo y se sometió a técnicas matemáticas de tipo estadístico, señalando los parámetros de posición o de dispersión que se utilizaron, de la información que se obtuvo a través de las fuentes que se investigó a través de la entrevista, encuesta u observación.

\section{Herramienta utilizada}

Para la elaboración del plan de marketing del caso propuesto, se utilizó la herramienta. Marketing estratégico pasó a paso.

\section{Resultados}

La herramienta metodología utilizada - Marketing estratégico paso a paso- ha sido difundida y Aplicada a MYPIMES y a grandes empresas, sin embargo, no se conoce de estudios realizados para negocios de la rama de distribución de pintura, por lo que luego de culminado el mismo se considera como una herramienta que facilita el conocimiento de las oportunidades de mercado, dando prioridades a los consumidores y analizando muy de cerca a la competencia, alejándose del producto para poner mayor énfasis en la búsqueda de un valor agregado al producto que se revierta en un aporte al consumidor. 
De igual forma en el momento de valorar las oportunidades de mercado, es importante conocer la evolución y la tendencia del mismo con una idea clara del ámbito de la actividad en el que se mueve la empresa o negocio, así como, de los espacios en las que no debe estar.

Por otro lado los consumidores son los factores claves que se debe considerar dentro del plan de marketing, y la mejor manera de tener un conocimiento aceptado acerca de ellos es mediante un proceso de segmentación, para ello fue necesarios agrupar en función a variables homogéneas y diferenciándoles con respecto a otros grupos de consumidores.

Además esta herramienta nos permite conocer a profundidad a la competencia, partiendo desde su evolución, objetivos, ventajas y desventajas que tienen los competidores.

Bajo esta perspectiva los resultados obtenidos luego de todo el proceso se sintetizan en las siguientes cuestiones:

\section{- ¿Cuáles son los aspectos administrativos del negocio de mayor incidencia en el bajo nivel de ventas?}

La administración del negocio se centra únicamente en la gerencia, sin dar información de procesos, control interno, al personal que labora dentro de la institución. Se realizará un organigrama lineal institucional, para que cada uno de los que trabajan, conozcan los deberes y responsabilidades que les corresponde, ya sea esta individual o en forma colectiva.

Los propietarios que validaron la propuesta tienen amplia trayectoria en administración, conocen sus responsabilidades y deberes, han participado activamente en los principales logros y organización del negocio; las debilidades en la administración de los recursos son de orden técnico, lo que guarda relación con los resultados de las encuestas y entrevistas.

\section{- ¿Cuáles son los procesos actuales de comercialización?}

La administración de los procesos de comercialización se realiza utilizando los datos aislados de los diferentes programas informáticos, no existe aprovechamiento de la base de datos; la información se halla dispersa y conforme a la costumbre, los responsables de la oficina evalúan el proceso de comercialización únicamente en base a facturas a mano, no se utilizan los sistemas informáticos, esto dificulta mantener un registro de cada cliente con su propio código. Se comprará un sistema informático, para que ayude al negocio a tener información clara y precisa de los clientes y proveedores.

\section{- ¿Cómo mejorar el desempeño de los empleados?}


Adicionalmente el sistema informático, con los programas de decoraciones de viviendas no guarda relación con el personal que labora dentro del negocio, su preparación es deficiente en lo que respecta a las normas de atención a clientes, dificultando incursionar a corto plazo en venderotras marcas de pinturas, que se comercializan en el mercado. Se fomentará cursos de capacitación sobre ventas y atención al público en general, para los empleados en forma individual, y sobre todo los cursos del FEN-SUI o decoración de colores, de acuerdo a las edades, sexo y carácter.

\section{- ¿Analizar quiénes son competidores a los cuáles se enfrenta?}

En lo que respecta a los competidores, se identifican que los más fuertes son los que corresponden a las ferreterías, ya que estas, venden todos los productos para la vivienda; mientras que ARCO IRIS, vende únicamente pintura arquitectónica. Se tratará de ganar el mercado a los competidores mediante estrategias de mercado con: bonos, promociones y obsequios a los consumidores.

\section{- ¿Cómo se puede implantar un plan efectivo de ventas y cobros?}

En lo que se refiere al plan efectivo de ventas y cobros, lamentablemente es débil, ya que no cuenta con un programa informático, razón por la cual existen muchas de las veces pérdidas por parte de los dueños del negocio. Adicionalmente el plan de marketing es importante para añadir: tendencias de crecimiento, volumen de ventas, inversiones más altas, mayor utilidad para los propietarios del negocio.

\section{- ¿Cuáles serán los requisitos indispensables para establecer y poner en marcha un plan eficiente de promoción de productos en el negocio?}

Los dueños del negocio, opinan que lo primero que deben realizar son conversaciones directas con los propietarios de las marcas de pinturas que venden dentro del negocio, y que a través de la entrega de folletos, gorras, camisetas y publicidad, se puedan poner en marcha el plan de promoción de productos que se puede realizar con descuentos directos en las pinturas, o con entrega de obsequios que serán entregados por parte de los auspiciantes principales del negocio ARCO IRIS. El propietario del negocio considera que el sistema del nuevo plan de promoción de productos adicionalmente garantizará la integridad de la entrega de obsequios a los clientes y consumidores finales.

\section{Referencias Bibliográficas}

Jimenes C, W. (2004). Introducción al estudio de la teoría administrativa. México: FCE. 
Sampieri, R., Fernández, C., \& Bautista, P. (2010). Metodología de la Investigación. México: McGRAW-HILL/INTERAMERICANA EDITORES. S.A. DE C.V.

Servicio de Rentas Internas (SRI). (3 de Mayo de 2016). ¿Qué son las PYMES? Obtenido de www.sri.gob.ec: www.sri.gob.ec/de/32

Terry, G. (2005). Principios de Adminsitración. México: Continental.

Quevedo Vázquez, J. O. (2013). Plan de marketing para mejorar los procesos de comercialización para el almacén Arco Iris de Azogues. 